\title{
Synchronous Distance Education Via the Internet
}

\author{
J. Mark Pullen, George Mason University \\ Department of Computer Science and C3I Center \\ George Mason University \\ Fairfax, VA 22030
}

\begin{abstract}
This paper describes a vision for networked distance education and ongoing experiments intended to increase our understanding of how distance education can be conducted most successfully at the university level.

Our vision includes the relationship between delivery media and modes in the future learning environment, the role of information technologies in scholarship, and the benefits of unlimited access to global information resources. A special concern here is effective use of various media in distance education. For example the traditional role of video delivery is analyzed and seen to be, in many cases, more a matter of past necessity than effective future delivery.

In support of our conclusions a series of experiments conducted at GMU are described. These have involved teaching real, for-credit courses to remote students using the Internet. While many universities are experimenting with asynchronous delivery via email, web, and related technologies, our work is unusual in that it involves synchronous delivery. The most enlightening of our experiments involved use of the Internet multicast backbone (Mbone) and its associated suite of multimedia tools. Ongoing experiments involve use of commercial off-the-shelf multimedia with low-cost workstations and commercial Internet service.
\end{abstract}

\section{A vision for university distance education}

The impact of Global Information Infrastructure or GII (of which the Internet is the current manifestation) will nowhere be greater than in higher education. The possibility to offer courses anytime and anywhere will result in a mix of on-campus, remote attendance at live courses, and recorded multimedia courses. Such courses will have to be tailored to distance delivery modes appropriate to their contents and the needs of their students: broadcast to mass audiences, remote delivery to groups gathered off campus, and delivery to electronic desktops in individual homes and offices as well as traditional campuses. Publication and course materials will use electronic multimedia to a much greater degree. Students will be able to work in teams regardless of any distance that may separate them. They will be able to learn using global information resources, distributed faculty, and experts from industry and government. This brave new future will require that universities pursue new, more effective models of teaching that are enabled by the technologies [1].

We now have technologies at our disposal that are capable of supporting new relationships with communities on-campus, regionally, and beyond. It is clear that such technologies will become even better and less expensive over time. Of particular interest for distance learning are emerging multimedia telecomputing ranging from email and netnews to web, audio, and video. These technologies are more effective when combined skillfully with traditional face to face and paper-based communication options. At the same time the universities' customers (students and their sponsors, especially employers) are learning that they can be demanding consumers. For the first time it has become economically feasible to distribute classes to individuals at home or work without capturing in its entirety a mass broadcast medium such as a cable TV channel. Soon there will be competition to deliver instruction where it is most productive for the student: in the home, office, or in the classroom.

Another major role of academia has been research and related doctoral education. The rule has been "publish or perish." This is not likely to change, but the medium of publication will change. An option will be to "publish" via the GII. Journals, peer selection and editorial boards will remain, but paper publications increasingly will become anachronisms, as the net is both faster and more current. A center of excellence in R\&D will count among its contributions to human knowledge the number of URLs with high-quality content it makes available on the net. Mastery of 
information technologies will hold a status coequal with mastery of spoken and written communication. This will not be an unmixed blessing to the cybertechnology experts, as they may find their skills in oversupply and competition fierce for a limited number of faculty spaces. But it will dictate that any learned institution must have a number of "cybertech gurus", just as today's university is not complete without an English department.

In some settings students learn more from each other than from the professor. The best professors always have taken advantage of this by emphasizing mentoring above lecturing. Future faculty will no longer be able to escape this phenomenon by retreating to arcane lectures, as the students will have many more information resources and will be considerably more sophisticated consumers. They will have available advanced collaboration technology that will enable them to learn from each other much more effectively. Faculty will be challenged to adapt their techniques accordingly. Options include "the professor as homepage editor" and "the student as teacher, the professor as critic". New information technologies in computing, databases, and networking will work inexorable change on universities. It is important to anticipate this and learn to use the new modes of teaching and learning.

We divide the technologies into three major categories. One-way media such as books, video tapes, cable and broadcast television deliver information without interaction, generally in an indiscriminate broadcast mode. They form the electronic equivalent of the mass lecture hall. Synchronous networked delivery media provide for interaction among instructor and students, and are capable of pinpointing the desktops of specific students. Asynchronous networked delivery media such as electronic mail and the World-Wide Web do not require that the provider and consumer of information participate at the same time. They support interaction but not immediate feedback. It is our expectation that successful distance education in the future will combine all of these modes (even lecture-hall style has a useful place under the right conditions).

\section{Distributed educational resources}

Until now a university has consisted of a collection of learning resources (reference material, faculty and laboratories) that was collocated and organized to permit systematic imparting of knowledge. The teaching institution of the future will consist of a collection of networked learning resources including reference material and faculty, in addition to remotely accessible experimental facilities enabled by computer simulation. University faculties of the future will not need to be collocated. In fact there will be real advantages to having a distributed faculty who live near objects of physical study and come together with students (and with each other) electronically. Also it will be practical for a single individual to serve on the faculty of several institutions, and for a single student to study with faculty from several institutions.

With the ability to communicate by video and voice over the GII, a major barrier to guest lectures is removed. An expert from anywhere in the world can address a class at any convenient time. Furthermore that class may consist of students connected via electronic desktops, also anywhere in the world. It will become practical to recruit a much wider variety of lecturers. If these lectures are minimally interactive in that on the average only one out of many students asks a question, it is possible for a world-class speaker to address hundreds or thousands of students. Also it becomes practical to assemble enough students from around the state, country or world to justify presenting a course on a topic of very limited interest.

Linking together libraries electronically is changing the nature of academic research. Literature searches that previously required hundreds of hours now may be accomplished in minutes. As libraries convert to electronic media and research journals move to online repositories it will also be possible to access through the GII most of the collected knowledge of humanity. One can expect to see students in elementary schools using primary sources such as the National Archives, while the need for a university to have at its physical core a research library will evaporate. In place of the library will come a need for skill and understanding in network-based research.

At the leading edge of the distributed university is the distributed research team. Already researchers in different institutions are collaborating over the Internet, to a degree that previously was impossible due to physical separation and slow postal service. This trend can be expected to continue to a point where work in science, engineering, the humanities, and even the arts is performed by highly fluid communities of intellectuals who meet in person rarely only on rare occasions. This in turn will accelerate development of distributed teaching communities that grow out of the distributed research communities. 


\section{Networked multimedia in distance education}

At George Mason University (GMU) we have begun to investigate a range of multimedia tools and means of communications that will support the new models of distance education, in preparation for the impending revolution. We expect that ultimately desktop delivery in the student's home or workplace will be one of the most important modes. The area in which we are focusing is synchronous networked systems, as these will also support the other two modes (one-way and asynchronous networked). We have found a variety of technologies holding great promise, but in most cases they lack the robustness and level of integration needed to support distance education. In this regard it is important to remember that faculty experimenting with alternative delivery methods must still focus on providing an effective learning experience for the student. Thus technologies that are too fragile or do not comprise an integrated learning environment cannot be considered. In a recent experiment the best capability we found to meet our needs was the Internet Multicast Backbone (Mbone) with its available suite of session directory, audio, video, whiteboard, and recording tools[2], loaded on a multimedia Unix workstation.

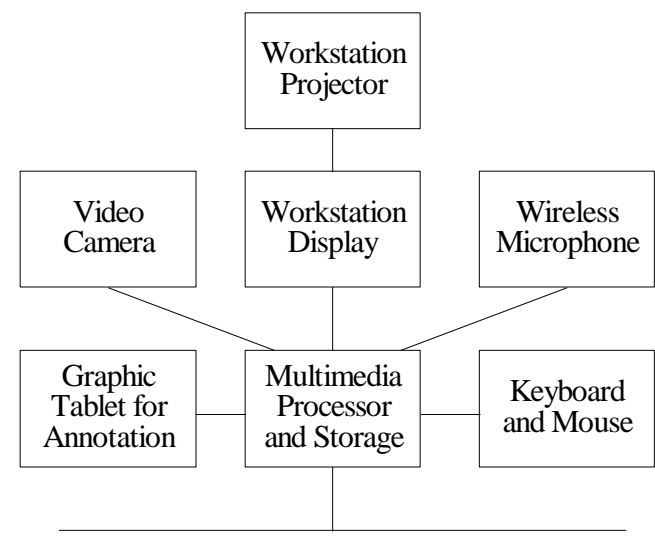

LAN with Mbone Support

Figure 1. Multimedia Instructor Workstation

To gain an effective understanding of the issues involved it was necessary actually to deliver a course with remote students. The pilot course was a Senior Computer Science elective, Computer Networking Systems. Because multimedia networking is a topic of the course, the instructor and students in this course held more curiosity about the means of delivery than others would have. This level of engagement proved valuable as we found that a great deal of energy, flexibility, and patience is needed for such an activity. This was in spite of the advantage we enjoy from our location in the high-technology region of Northern Virginia with many employed students who have access to the Internet. (Not coincidentally, our area has vehicular traffic jams of legendary proportions. Because GMU has many students who commute from offcampus, we had both the motivation and the opportunity to make early trials of Internet desktop distance education.)

We began by analyzing the media needed for teaching. First and foremost is audio; in many teaching situations the spoken voice carries nearly all of the information. Beyond audio are video, for moving images, and graphics, for still images. For best effect it must be possible to annotate the graphics spontaneously and have the annotation appear on the student's display in real time. Also a channel coming back from the student is needed, capable of spoken or written questions. Without such an interactive capability we find much of the potential of distance education is lost. The stimulus of immediate questions during a class helps focus the students, keep up with current aspects of material, and serve as a check against accidental omissions by the instructor. Of course asynchronous distance education tools such as electronic mail, Web pages, and in our case an integrated network simulation are also highly valuable. However we have found these to be no substitute for real-time interaction as part of a quality learning experience [3].

A well-established model of distance learning on our campus and many others is delivery by television. One result of television's well-established position is that faculty considering distance education generally assume that video is the most important component. It is certainly true that video has served as a surrogate for graphics. However with both video and whiteboard available in the Mbone tools, it became evident quickly that most of the information needed by the student is in the whiteboard graphics. In order to support the remote learning process effectively, it was necessary to create in the neighborhood of forty slides per class session. Mbone video is of relatively low quality a few frames per second, with occasional anomalies due to packet loss. Thus one might be tempted to believe that higher quality video would displace graphics. This is indeed possible, but only at great cost. The data load to download the slides and synchronize their display averages under one kilobit per second over the whole class, whereas a video signal of equivalent resolution would require several megabits per second even with compression, and therefore would not be cost-effective. 
Certainly video does have a role in the distance education environment. In many subjects, pre-recorded video segments are available to enhance the classroom experience. These can apply to distant students as well as in the classroom. However, pre-recorded video need not be distributed in real time. It can be distributed in advance either on recorded media such as CD-ROM, or by previous network transmission during gaps in realtime traffic. It is also true that some subjects such as drama or public speaking may actually demand realtime video for full effectiveness. However it is our experience that, for typical course delivery, real-time video transmission of a "talking head" is not a costeffective use of network capacity.

\section{Synchronous course delivery via Internet}

After a semester of experimentation with "remote" students in the adjoining laboratory, we launched the real thing. Critical to our success was finding students of an experimental bent. Our two volunteers worked 15 miles away at a facility in Alexandria, Virginia. One of the two alternated between attending class at GMU and remotely, the other did not come to campus at all. The Mbone software configuration on Sun workstations with T1 Internet support was used at both locations. Slides were edited for each class and stored on-line in the laboratory. As a hedge against network or equipment problems each lecture was recorded on disk. This was providential as outages did occur, and also was popular with students attending locally as a way to make up a missed class.

Two students were too few for statistical significance and there was an obvious Hawthorne effect from participation in the experiment. However the highly positive reports from the experimental students appear to have considerable validity. They reported that the learning environment was at least equal to a regular classroom, in fact the student who participated both remotely and in the classroom feels strongly that the remote form is better. Remote students can see the slides better, receive the instructor's voice (captured using a wireless microphone) with no background noise, and receive full attention to their questions. The only drawback other than occasional equipment and network problems was the need to coordinate a proctor for exams at the remote location.

\section{The next generation}

Our work in technologies and pedagogy for distance education is continuing. The strength of the
Mbone tools is their integration and completeness; the weakness is lack of commercial support and need to be connected to the Mbone, which generally is impractical for students at home. We have demonstrated a similar capability to use dial-in Internet providers with commercial graphic and audio software, without video. This capability was tested with volunteer students at home during several class sessions and found to be effective. The most significant problem experienced was occasional loss of Internet connectivity, which we expect will be overcome by a new direct link between a service provider and our campus backbone.

We expect to add to the dial-up configuration a "snapshot" video capability. On this basis we will be seeking larger numbers of students to participate in classes via Internet dial-in from home in Fall 1996. Ultimately we expect to expand from regularly scheduled courses to professional education, where graduate-level courses are delivered to office electronic desktops. We believe short courses can be distributed successfully in this form within the next two years.

\section{Conclusions}

The impact of the emerging Global Information Infrastructure on education cannot be avoided. At all levels: pre-college, undergraduate, Master's and professional courses, and doctoral-level research, major changes will be felt. The time is at hand to experiment broadly with all media and modes of distance education. Undoubtedly we have much to learn about matching the needs of various subjects and learning communities with technological solutions. We are moving toward a future, not far away, when anyone, anywhere, will be able to choose from the broadest possible range of learning opportunities without regard to their location.

\section{References}

1. Hunter, B. "Internetworking: Coordinating Technology for Systemic Reform", Communications of the ACM, May 1993

2. Macedonia, M.R. and D.R. Brutzman, "Mbone Provides Audio and Video Across the Internet", IEEE Computer, April 1994

3. Pullen, J.M. "Prototyping the Classroom of the Future", Symposium on Rapid Systems Virtual Prototyping, Applied Physics Laboratory, Johns Hopkins University, May 1995 